\title{
Anti-allergic action of bacillus Calmette-Guerin extract in experimental mast cell-mediated anaphylactic models
}

\author{
MINGLI SUN ${ }^{1}$, YONG WANG $^{1,2}$, HAISHAN ZHAO ${ }^{1}$, WEIFAN YAO ${ }^{1}$ and XIAOSONG YU $^{2}$ \\ ${ }^{1}$ Department of Pharmacology, School of Pharmacy, China Medical University, Shenyang, Liaoning 110122; \\ ${ }^{2}$ Department of General Practice, The First Hospital of China Medical University, Shenyang, Liaoning 110001, P.R. China
}

Received May 9, 2016; Accepted April 4, 2017

DOI: $10.3892 / \mathrm{mmr} .2017 .7383$

\begin{abstract}
Allergy is an acquired hypersensitivity reaction of the immune system mediated by IgE-induced mast cell degranulation. In China, bacillus Calmette-Guerin extract (BCGE) has been shown to be clinically effective for regulating immunity, which enhances the resistance of the body to anaphylactic disease, infectious diseases and cancer. However, the mechanisms remain to be fully elucidated. The present study investigated the potential anti-allergic effects of BCGE in animal models of mast cell-dependent anaphylaxis and mechanisms of BCGE in mast cells. Anti-allergic actions of BCGE were evaluated in passive cutaneous anaphylaxis, dextran T40-induced scratching behavior mouse models, and in ovalbumin (OVA)-induced contraction of intestinal tube isolated from OVA-sensitized guinea pigs. Direct mast cell-stabilizing effects of BCGE were examined in mast cells from the abdominal cavity of OVA-sensitized rats. Anti-allergic signaling mechanisms of BCGE in mast cells were investigated by detection of cyclic adenosine monophosphate levels and protein kinase A expression in mast cells. It was observed that BCGE prevented OVA-induced cutaneous vascular hyperpermeability, skin itching, elevation in plasma histamine levels and abdominal cavity fluid mast cell degranulation in animal models, in a dose-dependent manner. BCGE also suppressed OVA-mediated guinea pig intestinal tube contraction in vitro. In addition, BCGE was found to increase the levels of interferon- $\gamma$, and reduce the levels of interleukin- 4 and OVA-sIg E levels in OVA-sensitized rats. BCGE also increased levels of cyclic adenosine monophosphate and the expression of protein kinase A in mast cells separated from the abdominal cavity fluid of OVA-sensitized rats. In conclusion, the results suggested that BCGE possesses anti-allergic
\end{abstract}

Correspondence to: Dr Xiaosong Yu, Department of General Practice, The First Hospital of China Medical University, 155 Nanjing Street, Heping, Shenyang, Liaoning 110001, P.R. China E-mail:xsyu@mail.cmu.edu.cn

Key words: bacillus Calmette-Guerin extract, passive cutaneous anaphylaxis, mast cell degranulation, cyclic adenosine monophosphate, protein kinase $\mathrm{A}$ activity by inhibiting IgE-induced mast cell degranulation, providing a foundation for the development of BCGE for the treatment of mast cell-mediated allergic disorders.

\section{Introduction}

Allergy is an acquired hypersensitivity reaction of the immune system in response to normally innocuous environmental substances, which manifests in several forms ranging from minor urticaria, allergic rhinitis and conjunctivitis, and asthma to life-threatening anaphylaxis $(1,2)$, causing a substantial socio-economic burden. Several therapeutic trials have been performed to modulate allergy, however, with limited success. These have included the prevention of Th2 responses, enhancement of Th1 responses and decreasing of IgE concentrations (3). Glucocorticoids are important and effective for the treatment of anaphylactic disease. However, it is well known that the prolonged use of high doses of glucocorticoids causes a range of side effects (4). Therefore, the development of novel and effective therapies for anaphylactic disease is required.

Mast cells are important in allergic responses. An allergic response is triggered by allergen sensitization, which elicits Th2 immune responses, including an increase in levels of interleukin (IL)-4 and IL-13, leading to immunoglobulin class switching in B cells to produce predominantly $\operatorname{IgE}$ (5). Upon activation, mast cells undergo degranulation and release a range of biologically active substances, which are important in host defense and allergic reactions. Among the immune mediators released from the mast cells, histamine is one of the most well characterized and potent mediators $(1,6)$.

Bacillus Calmette-Guerin extract (BCGE), comprising predominantly lipopolysaccharide $(77.8 \%)$ and nucleic acids $(16.67 \%)$, is extracted from BCG using phenol. It has been shown that the polysaccharide in BCGE promotes the maturation of dendritic cells and the secretion of IL-12, which causes the transformation from $\mathrm{T} 0$ to $\mathrm{T} 1$ and the secretion of interferon (IFN)- $\gamma$ (7). A previous study indicated that nucleic acids from BCG can activate natural killer cells, enhances the production of IFN- $\gamma$ and IL-12, and inhibits the production of IgE (8-10). In China, BCGE has been shown to be clinically effective for regulating immunity, which enhances the resistance of the body to anaphylactic disease, infectious diseases and cancer (11-13).

However, the mechanisms remain to be fully elucidated. Previous studies demonstrated that BCGE was able to inhibit 
the production of IL-4 and IgE (14-16), and impede the transcriptional activity of nuclear factor- $\kappa \mathrm{B}(16)$. The present study aimed to investigate the effects and mechanisms of BCGE on IgE-mediated anaphylaxis using in vivo and in vitro models.

\section{Materials and methods}

Animals. A total of 60 female Kunming mice aged 6-8 weeks, weighing 18-22 g, 10 female Hartley guinea pigs aged 8-10 weeks, weighing 350-450 g, and 56 female Wistar rats aged 8-10 weeks, weighing 350-450 g, were purchased from the Center of Experimental Animals of China Medical University (Shenyang, China). All the animals were maintained under controlled conditions (temperature, $20-25^{\circ} \mathrm{C}$; humidity 40-70\%) with daily 12/12-h light/dark cycles. All the animals were provided with food and water ad libitum. All procedures were performed in accordance with the National Institutes of Health Guide for the Care and Use of Laboratory Animals (17).

All animal experiments were performed in accordance with the Institutional Animal Ethics Committee and Animal Care Guidelines for Experimental Animals of China Medical University.

Ovalbumin (OVA)-induced passive cutaneous anaphylaxis $(P C A)$. The rats $(n=8)$ were sensitized by intramuscular injection of OVA (10 mg/kg; Sigma-Aldrich; Merck KGaA, Darmstadt, Germany) and DPT vaccine ( $2 \mathrm{ml}$ per rat; Chengdu Institute of Biological Products Co., Ltd., Chengdu, China). Following sensitization for 14 days, the whole blood samples were obtained and placed at room temperature for $2 \mathrm{~h}$, then were centrifuged at $1,000 \mathrm{x}$ g for $20 \mathrm{~min}$ at room temperature, and serum was separated and mixed; following which intradermal injection of another 48 rats was performed using three titers $(1: 2,1: 8$ and 1:32) in every rat $(0.1 \mathrm{ml}$ at each titer). At $24 \mathrm{~h}$ post-injection of sensitized serum (three titers/rat, $0.1 \mathrm{ml}$ each titer), the rats were challenged by an intravenous injection of a $1 \mathrm{ml}$ mixture of Evan's blue and OVA (10 mg OVA; $5 \mathrm{mg}$ Evan's blue). BCGE (0.026, 0.077 and $0.232 \mathrm{mg} / \mathrm{kg}$; Zhejiang Pharmaceutical Co., Ltd., Zhejiang, China) or vehicle (normal saline; NS) was administered by intravenous injection every other day for 21 days prior to challenge. Evan's blue was extracted from the skin tissues in $5 \mathrm{ml}$ acetone and sodium chloride solution $(3: 7, \mathrm{~V} / \mathrm{V})$ for $48 \mathrm{~h}$ and measured using a spectrophotometer (WF2 W-2000; Unico, Dayton, NY, USA) at $590 \mathrm{~nm}$.

Dextran T40-induced scratching behavior. Scratching behavior was induced in the mice by intravenous injection of $1.25 \mathrm{mg} / \mathrm{kg}$ dextran T40. BCGE $(0.025,0.075$ and $0.225 \mathrm{mg} / \mathrm{kg})$ or vehicle (NS) was administered by intravenous injection every other day for 21 days prior to treatment with dextran T40. Dextran T40 was administered $1 \mathrm{~h}$ following the final administration of BCGE or NS. The scratching frequency and duration were detected within 30 min of induction by dextran T40. The anticoagulant whole blood samples were centrifuged at $1,000 \mathrm{x} \mathrm{g}$ for $15 \mathrm{~min}$ at room temperature and plasma was obtained. Histamine levels in plasma were also determined within 30 min of induction by dextran T40 using a histamine ELISA kit (cat. no. EA31; Oxford Biomedical Research, MI, USA).
OVA-induced intestinal tube contraction. The guinea pigs were actively sensitized using OVA, and the intestinal canal was prepared and placed into a Magnus bath with tyrode solution and $\mathrm{O}_{2}$ at $37^{\circ} \mathrm{C}$. Subsequently, one end of the intestinal canal was connected to a hook at the other end of the transducer with a $2 \mathrm{~g}$ load. The intestinal canal was preincubated with BCGE $\left(10^{-6}, 10^{-5}, 10^{-4}, 10^{-3}\right.$ and $\left.10^{-2} \mathrm{mg} / \mathrm{ml}\right)$ or vehicle control (tyrode) for $15 \mathrm{~min}$ prior to challenge with $1 \mathrm{mg} / \mathrm{ml}$ OVA. Contractions were monitored over a period of $30 \mathrm{~min}$.

Measurement of cytokine and OVA-sIg E levels. The rats were sensitized by intramuscular injection of OVA $(10 \mathrm{mg} / \mathrm{kg})$ and a DPT vaccine ( $2 \mathrm{ml}$ per rat). BCGE (0.026, 0.077 and $0.232 \mathrm{mg} / \mathrm{kg}$ ) or vehicle (NS) was administered by intravenous injection 7 days prior to sensitization every other day for 21 days. On days 7, 14 and 21 post-sensitization, the whole blood samples were obtained and placed at room temperature for $2 \mathrm{~h}$, then were centrifuged at $1,000 \mathrm{x}$ g for $20 \mathrm{~min}$ at room temperature, and rat serum was collected for detection of the levels of IL-4 and IFN- $\gamma$ using an ELISA kit (cat. nos. R4000 and RIF00; R\&D Systems, Inc., Minneapolis, MN, USA) according to the manufacturer's protocol. The level of OVA-s IgE in the serum was detected on the day 14 post-sensitization using an ELISA kit (cat. no. E110-117; Bethyl Laboratories, Inc., Montgomery, TX, USA) according to the manufacturer's protocol.

OVA-induced mast cell degranulation. On day 14 following sensitization by OVA, the rats were sacrificed and $10 \mathrm{ml} \mathrm{Hank's}$ solution was injected into the abdominal cavity. Following gentle massaging of the abdomen for $3 \mathrm{~min}$, the abdominal cavity was opened and peritoneal fluid was collected. The peritoneal fluid was placed on ice for $10 \mathrm{~min}$, centrifuged at $120 \mathrm{x}$ g for $5 \mathrm{~min}$ at $4^{\circ} \mathrm{C}$, and the supernatant discarded. The cells were suspended in Hank's solution $\left(2 \times 10^{5} / \mathrm{ml}\right.$. The mast cell suspension was placed into a tube containing BCGE $\left(10^{-6}\right.$, $10^{-5}, 10^{-4}, 10^{-3}$ and $10^{-2} \mathrm{mg} / \mathrm{ml}$ ) or vehicle control (Hank's solution) and OVA for $5 \mathrm{~min}$ at $37^{\circ} \mathrm{C}$. The reaction was terminated on ice, and the mast cell solution was placed onto a slide treated with $0.025 \%$ neutral red ethanol solution to stain for $3 \mathrm{~min}$. Following staining, 100 mast cells were selected at random for calculating the percentage of degranulation.

Measurement of cyclic adenosine monophosphate (cAMP) levels in mast cells. The mast cell suspension was prepared as described above, and was placed into a tube containing BCGE $\left(10^{-6}, 10^{-5}, 10^{-4}, 10^{-3}\right.$ and $\left.10^{-2} \mathrm{mg} / \mathrm{ml}\right)$ or vehicle control (Hank's solution) and OVA for $10 \mathrm{~min}$ at $37^{\circ} \mathrm{C}$, with the reaction terminated using frozen acidified ethanol. The mixture was then placed in liquid nitrogen for $4 \mathrm{~min}$ and melted in room temperature, which was repeated five times and followed by centrifugation at $120 \mathrm{x}$ g for $5 \mathrm{~min}$ at $4^{\circ} \mathrm{C}$. The supernatant was collected $(0.9 \mathrm{ml})$, unwatered by decompression and then dissolved in $1 \mathrm{ml}$ PBS. The level of cAMP was detected using a radioimmunoassay kit according to the manufacturer's protocol.

Western blot analysis. Proteins were extracted from the mast cells treated as above using a Nuclear and Cytoplasmic Protein Extraction kit (Beyotime Institute of Biotechnology, Inc., Haimen, China). The extracts were boiled for $5 \mathrm{~min}$ with 
A

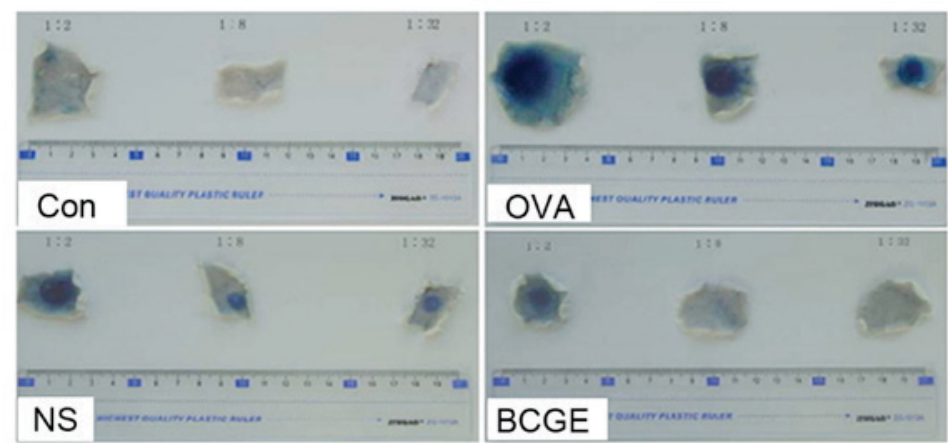

B

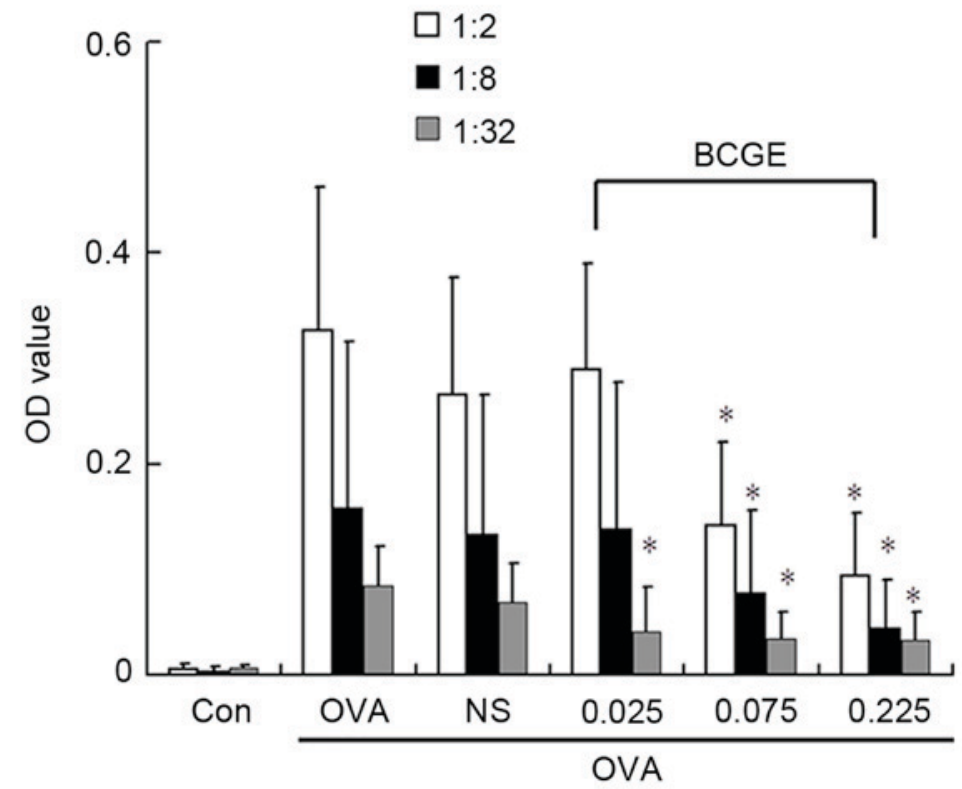

Figure 1. Effects of BCGE on PCA induced by OVA. (A) OVA challenge induced localized vascular hyperpermeability and plasma extravasation on Evan's blue staining, visualized as intense blue staining in the skin. Photomicrographs shown are representatives of three separate experiments with three serum titers (1:2, 1:8 and 1:32) in every rat. The image for BCGE was with a concentration of $0.225 \mathrm{mg} / \mathrm{kg}$. (B) BCGE dose-dependently inhibited OVA-induced plasma extravasation. Values are expressed as the mean \pm standard deviation with three mice per treatment group. * P<0.05, vs. NS control. Con, negative control; OVA, ovalbumin (positive control); NS, normal saline (vehicle control); BCGE, bacillus Calmette-Guerin extract; OD, optical density.

loading buffer. The protein concentration was determined using a bicinchoninic protein assay kit (Beyotime Institute of Biotechnology). For western blotting analysis, an equal quantity of total protein $(30 \mu \mathrm{g} / \mathrm{lane})$ was loaded, and separated by sodium dodecyl sulfate polyacrylamide gel electrophoresis on a $12 \%$ gel and transferred onto polyvinylidene difluoride membranes. The membranes were washed with PBST and 5\% skim milk for $1 \mathrm{~h}$ at room temperature. Following three washes with PBST, the membranes were incubated with primary antibodies against protein kinase A (PKA; cat. no. ab211265; 1:1,000 dilution) and GAPDH (cat. no. ab9485; 1:2,500 dilution; Abcam, Cambridge, UK) at $4{ }^{\circ} \mathrm{C}$ overnight. Following three further washes, the blots were subsequently incubated with a with horseradish peroxidase-conjugated secondary antibody (cat. no. E030120-02; dilution, 1:2,000; EarthOx Life Sciences, Millbrae, CA USA) for $1 \mathrm{~h}$ at room temperature. Following three final washes, the blots were visualized using Beyo ECL Plus reagent (Beyotime Institute of Biotechnology), according to the manufacturer's protocol. Densitometric analysis of the western blots was achieved using ImageJ v1.48 software (National Institutes of Health, Bethesda, MD, USA).
Statistical analysis. Statistical analyses were performed using SPSS 16.0 software (SPSS, Inc., Chicago, IL, USA). Data are presented as the mean \pm standard deviation. One-way analysis of variance followed by Student-Newman-Keuls test was used to determine significant differences between treatment groups. $\mathrm{P}<0.05$ was considered to indicate a statistically significant difference.

\section{Results}

BCGE suppresses OVA-induced PCA. The rats were sensitized with an intradermal injection of serum from OVA-sensitized rats and challenged with intravenous OVA, After 30 min, Evan's blue dye resulted in intense blue staining at the intradermal injection sites, compared with the control (Fig. 1A). BCGE $(0.026,0.077$ and $0.232 \mathrm{mg} / \mathrm{kg})$ significantly suppressed OVA-induced PCA vascular permeability $(\mathrm{P}<0.05)$ in a dose-dependent manner, compared with the NS control (Fig. 1A and B). Taken together, these data indicated that BCGE was able to suppress mast cell-mediated anaphylactic reactions in vivo. 
A

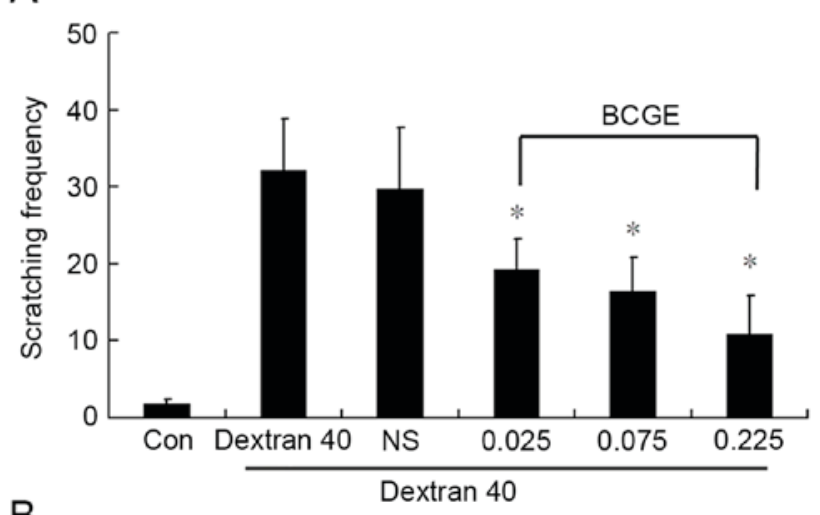

B

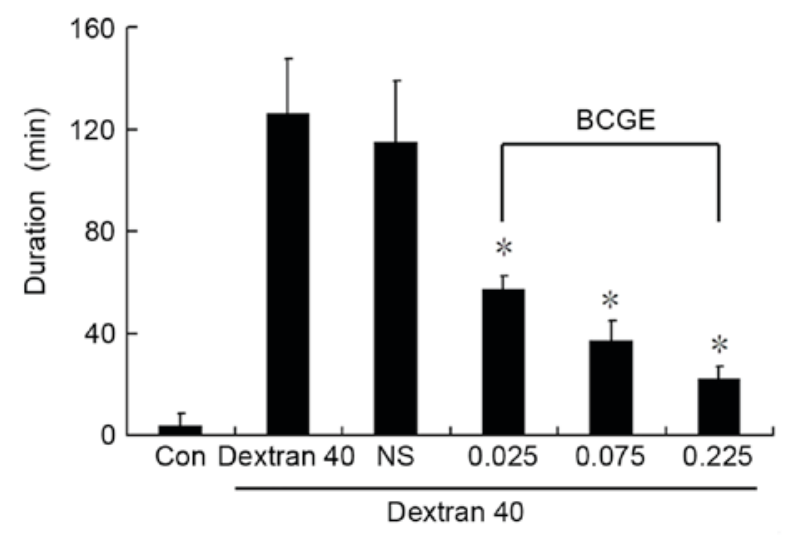

C

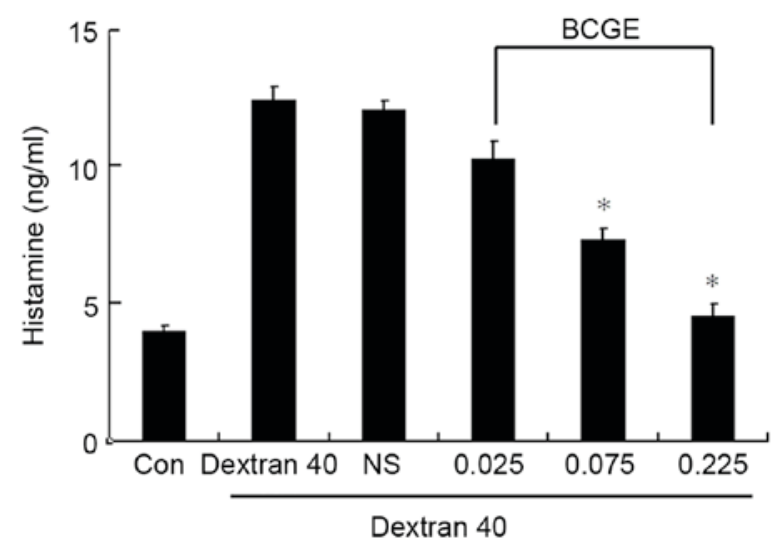

Figure 2. Effects of BCGE on scratching behavior induced by dextran T40. Skin itching of mice was induced by dextran T40, and (A) scratching frequency and (B) duration were detected within 30 min of sensitization by dextran T40. (C) Plasma histamine levels were determined 30 min following dextran T40 induction. Values are expressed as the mean \pm standard deviation with three mice per treatment group. ${ }^{*} \mathrm{P}<0.05$, vs. NS control. Con, control; NS, normal saline; BCGE, bacillus Calmette-Guerin extract.

BCGE suppresses scratching behavior induced by dextran T40. The mice treated with dextran T40 developed significant scratching behavior and the duration of scratching behavior was prolonged within the $30 \mathrm{~min}$ period (Fig. $2 \mathrm{~A}$ and B), BCGE pretreatment $(0.026,0.077$ and $0.232 \mathrm{mg} / \mathrm{kg})$ significantly prevented scratching behavior in a dose-dependent manner, compared with the NS control. In addition, plasma histamine levels were significantly increased following treatment with dextran T40, whereas BCGE caused a dose-dependent suppression of the rise in plasma histamine levels, compared

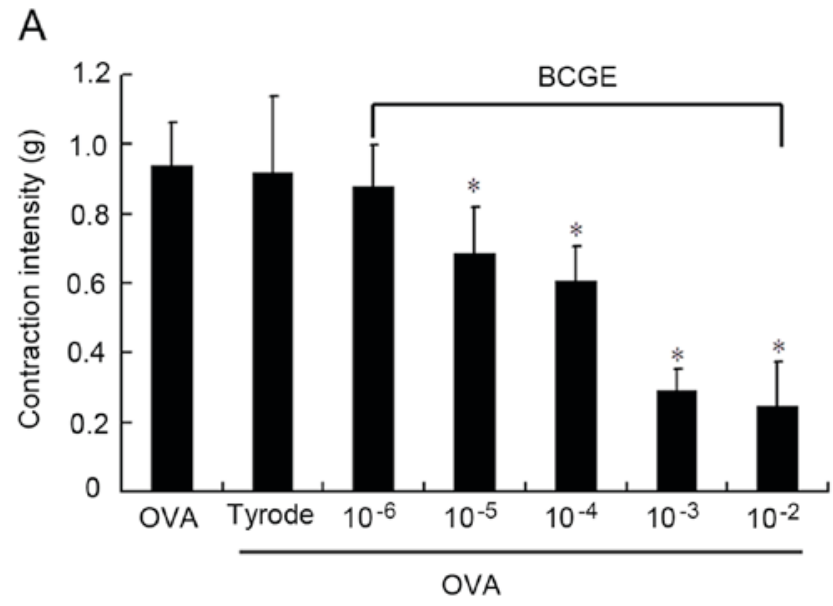

B

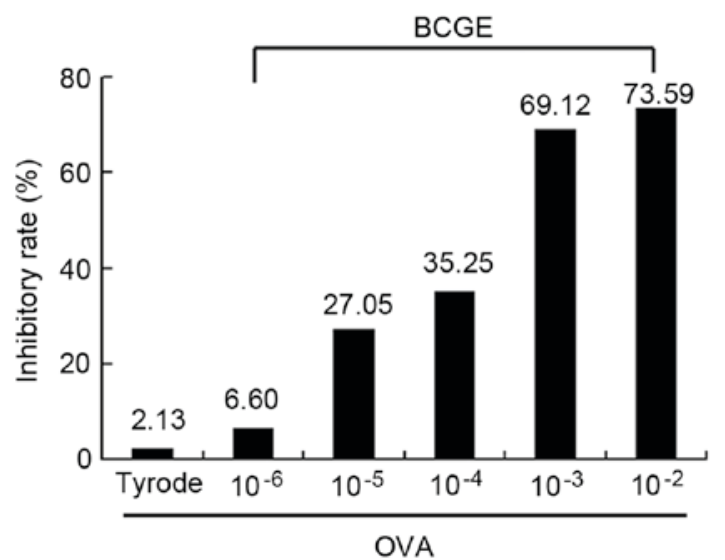

Figure 3. Effects of BCGE on OVA-induced anaphylactic intestinal tube contraction of guinea pigs. (A) Concentration-course evaluation of BCGE on OVA-induced anaphylactic intestinal tube contraction. (B) Inhibitory rate of BCGE on OVA-induced anaphylactic intestinal tube contraction within $30 \mathrm{~min}$. Values are expressed as the mean \pm standard deviation of three separate experiments. ${ }^{*} \mathrm{P}<0.05$, vs. tyrode control. OVA, ovalbumin; BCGE, bacillus Calmette-Guerin extract.

with the NS control (Fig. 2C). These results suggested that BCGE inhibited mast cell-mediated anaphylactic reactions in vivo.

BCGE attenuates OVA-induced intestine anaphylactic contraction. OVA at a concentration of $1 \mathrm{mg} / \mathrm{ml}$ induced strong and sustained anaphylactic contractions of the sensitized guinea pig intestinal tube, reaching $0.919 \pm 0.22 \mathrm{~g}$ within $30 \mathrm{~min}$. BCGE pretreatment $\left(10^{-5}-10^{-2} \mathrm{mg} / \mathrm{ml}\right)$ significantly suppressed OVA-induced intestinal tube anaphylactic contraction, as reflected by the reduction in contraction intensity in a dose-dependent manner, compared with the tyrode solution control (Fig. 3A and B). These findings demonstrated the inhibitory effect of BCGE on inhibiting mast cell-mediated anaphylactic reactions in an in vitro model of anaphylaxis.

$B C G E$ regulates $O V A$-induced cytokine secretion and levels of OVA-sIg E. To obtain further insights into the molecular mechanisms involved in the effect of BCGE on mast cell-mediated anaphylaxis, the levels of IL- 4 and IFN- $\gamma$ were measured in the plasma of OVA-sensitized rats. The level of IL-4 increased significantly 7, 14 and 21 days following 
A

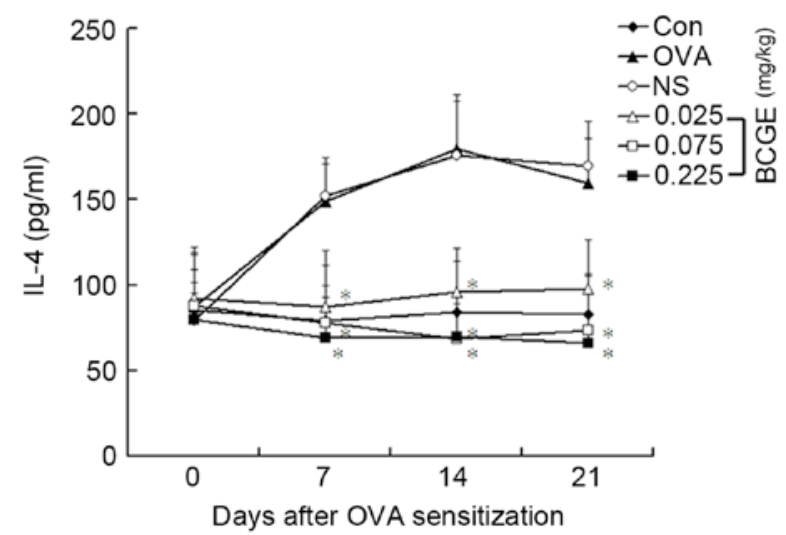

B

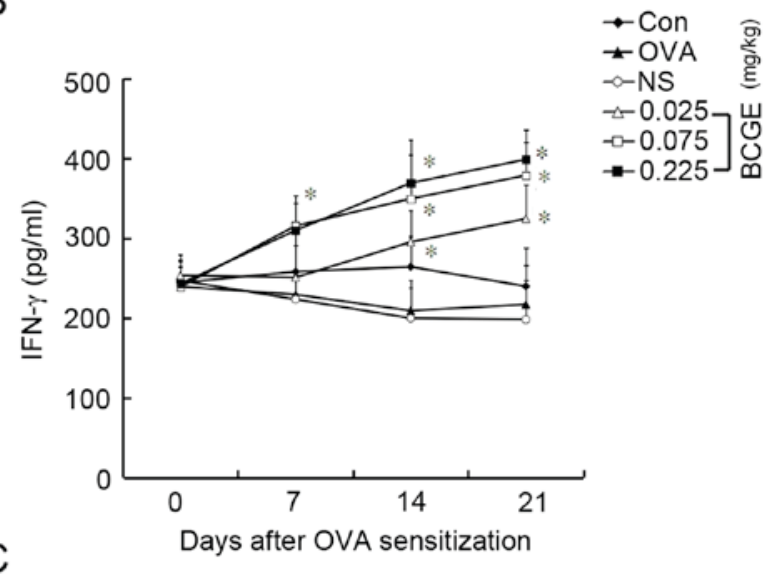

C

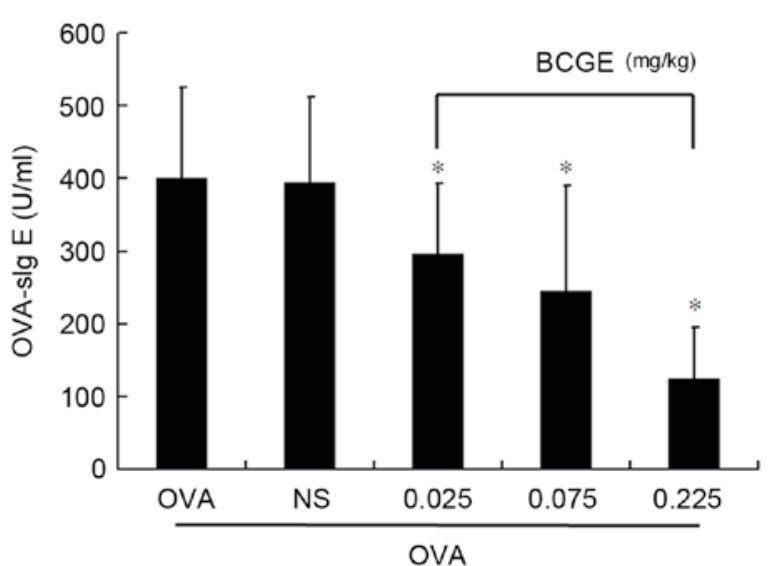

Figure 4. Effects of BCGE on levels of cytokines and OVA-sIg E in OVA-sensitized rats. Levels of (A) IL-4 and (B) IFN- $\gamma$ were measured in the plasma of OVA-sensitized rats 7, 14 and 21 days following sensitization by OVA. (C) OVA-sIg E was measured in the plasma of OVA-sensitized rats 21 days following sensitization by OVA. Values are expressed as the mean \pm standard deviation of three separate experiments. ${ }^{*} \mathrm{P}<0.05$, vs. NS control. OVA, ovalbumin; BCGE, bacillus Calmette-Guerin extract; Con, control; NS, normal saline; IL-4, interleukin-4; IFN- $\gamma$, interferon- $\gamma$.

sensitization $(\mathrm{P}<0.05)$, whereas no significant change in IFN- $\gamma$ was found. Intramuscular application of BCGE markedly reduced the OVA-induced increase in IL-4 $(\mathrm{P}<0.05)$, whereas the level of IFN- $\gamma$ increased in a dose-dependent manner (Fig. 4A and B). Furthermore, the intramuscular injection of BCGE significantly reduced the level of OVA-sIg E 21 days following sensitization (Fig. 4C).
BCGE suppresses OVA-induced mast cell degranulation. The mast cells stimulated by OVA showed significant degranulation, compared with the unstimulated mast cells. BCGE $\left(10^{-3}\right.$ and $10^{-2}$ ) significantly prevented OVA-induced mast cell degranulation in a concentration-dependent manner $(\mathrm{P}<0.05$; Fig. 5A and B). To elucidate the mechanism underlying the effect of BCGE on inhibiting OVA-induced immediate mast cell degranulation, the level of cAMP and the expression of PKA were determined upon OVA stimulation of the mast cells. The level of cAMP and expression of PKA were decreased significantly $10 \mathrm{~min}$ following stimulation by OVA. BCGE $\left(10^{-3}\right.$ and $\left.10^{-2}\right)$ significantly inhibited the OVA-induced decrease of cAMP and PKA in a concentration-dependent manner, compared with the vehicle control (Fig. 5C and D).

\section{Discussion}

IgE-induced mast cell degranulation is important in the development of allergic diseases. Mast cell-derived vasoactive mediators, including histamine, serotonin and peptidoleukotrienes, are rapidly released into the extracellular milieu $(18,19)$. Histamine appears to be most important to the manifestation of vasodilation, increased vascular permeability, pruritus and smooth muscle contraction in allergic anaphylaxis $(18,19)$. Strategies to prevent IgE-induced mast cell degranulation are central to the identification of drugs for the treatment of allergic diseases. For the first time, to the best of our knowledge, the present study demonstrated that BCGE possessed anti-allergic activity in mast cell-dependent in vivo and in vitro models of anaphylaxis. BCGE inhibited IgE-induced mast cell degranulation, likely via an increase in levels of cAMP levels and expression of PKA.

PCA and skin itching are commonly used in in vivo animal models to demonstrate IgE-mediated mast cell degranulation and to evaluate potential anti-allergy compounds $(18,19)$. PCA is a localized cutaneous allergic response, which results from allergen-induced vascular hyperpermeability and plasma extravasation, representing clinical features of urticaria. Skin itching is a generalized allergic reaction manifested as skin itching resembling the clinical features of anaphylaxis. Histamine, a preformed mediator rapidly released upon mast cell degranulation, is critical to anaphylaxis by binding to histamine receptors, leading to widening of intercellular gap junctions via endothelial cell retraction and vasodilation via increased production of nitric oxide $(20,21)$. In the present study, BCGE prevented OVA-induced cutaneous vascular hyperpermeability in rats and skin itching in mice, and caused elevation in levels of plasma histamine in mice. Allergen-induced anaphylactic intestinal smooth muscle contraction is a widely used in vitro model to demonstrate immediate mast cell degranulation and the rapid release of spasmogens, including histamine and peptidoleukotrienes, causing intestinal contraction. In the present study, BCGE significantly suppressed OVA-induced guinea pig intestine smooth muscle anaphylactic contraction over a $30 \mathrm{~min}$ interval.

To elucidate its anti-allergy mechanisms of action, the present study investigated the direct effects of BCGE on the degranulation of mast cells sensitized by OVA. BCGE significantly reduced the number of degranulated mast cells in a concentration-dependent manner. These findings indicated 
A

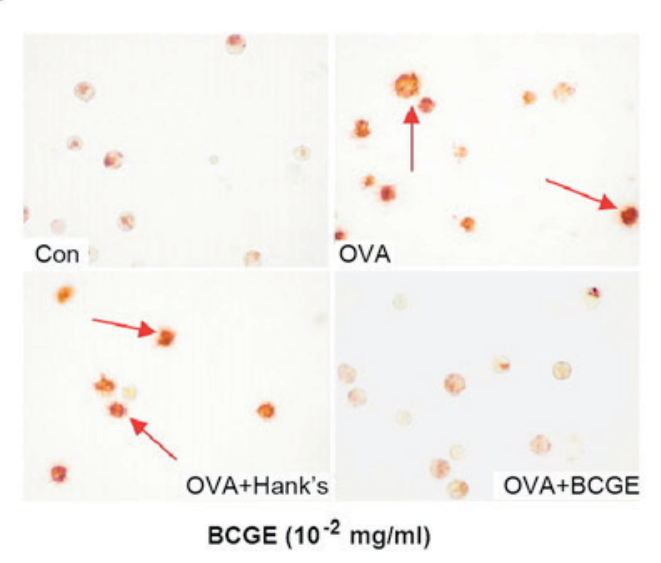

C

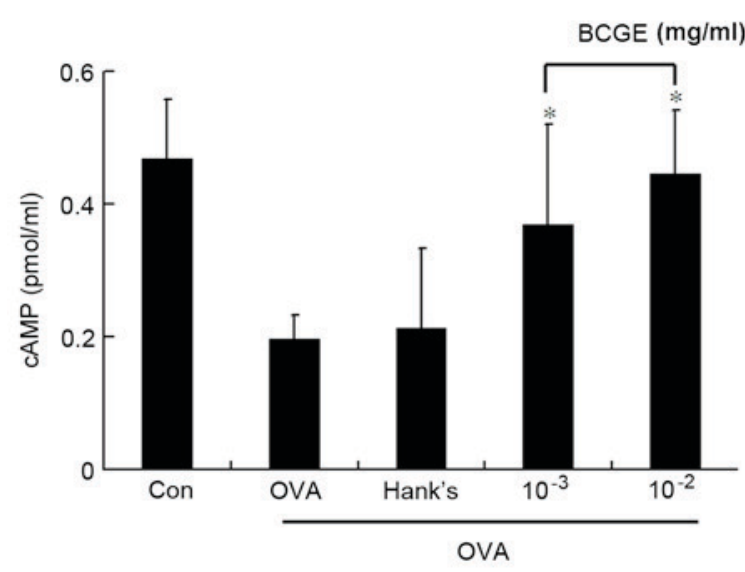

B

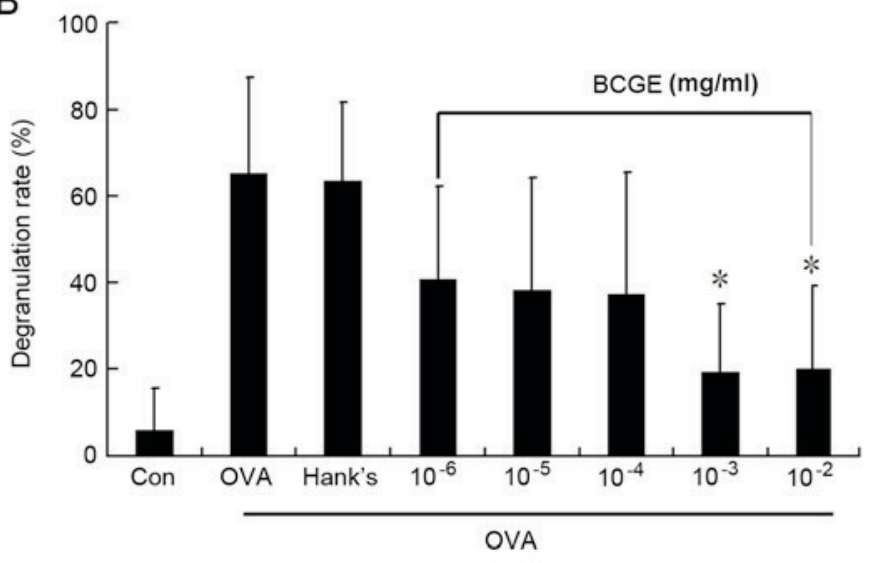

D
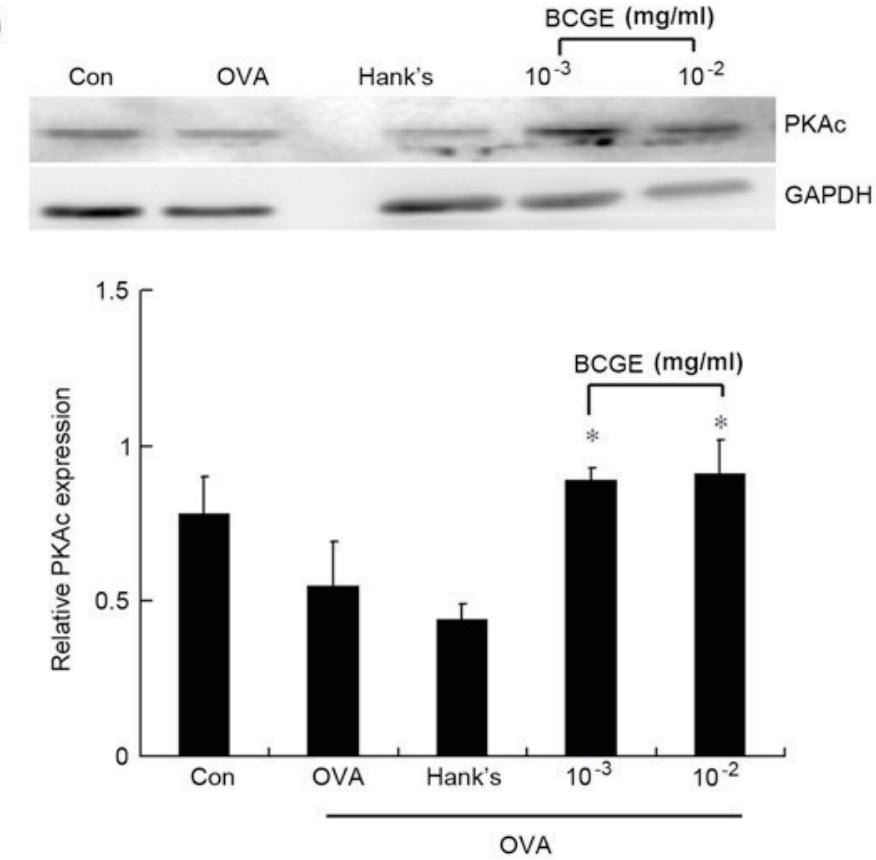

Figure 5. Effects of BCGE on OVA-induced mast cell degranulation. Mast cell degranulation was measured. (A) Photomicrographs of the Con, OVA, OVA+Hank's and OVA+ BCGE $\left(10^{-2}\right)$ groups are representative of three separate experiments, magnification, x20; red arrows indicate the degranulation of mast cell. (B) Data are expressed as a percentage of total cellular contents. (C) cAMP levels were detected using a radioimmunoassay kit according to the manufacturer's protocol. (D) Expression of PKA was detected using western blot analysis and immunoblots shown are representative of three separate experiments with similar patterns of results. Values are expressed as the mean \pm standard deviation of three separate experiments. "P<0.05, vs. Hank's. OVA, ovalbumin; BCGE, bacillus Calmette-Guerin extract; Con, negative control; cAMP, cyclic adenosine monophosphate; PKA, protein kinase A.

that BCGE inhibited OVA-induced mast cell degranulation. Mast cell activation and histamine release are tightly regulated by $\operatorname{IgE}$ from B cells, and serum levels of $\operatorname{IgE}$ are elevated in proportion to the development of allergic diseases (22-24). In the present study, OVA-induced total IgE levels in serum were reduced by BCGE. From these results, it was hypothesized that BCGE alleviated OVA-induced mast cell degranulation through the inhibition of $\mathrm{IgE}$ production. $\mathrm{IgE}$ synthesis by B cells is regulated by Th2 cytokines, particularly IL-4 and IL-13. In humans, the overproduction of IL-4 is a critical factor in allergic diseases. Sensitization to an allergen reflects the ability of an allergen to elicit a Th2 cell response, in which IL-4 and IL-13 drive the production of IgE by promoting class-switch recombination in B cells (1). In the results of the present study, BCGE decreased levels of IL-4 and IL-13, which are important in isotype switching to $\operatorname{IgE}$. These results indicated that BCGE reduced the serum levels of $\mathrm{IgE}$ by suppressing the Th2 response, particularly the production of IL-4 and IL-13.

The molecular mechanism underlying mast cell degranulation is a complex process by which several proteins are mediated. Each link is precisely controlled, with calcium, calmodulin, three phosphoinositide signaling pathways, two glycerol pathways and intracellular cyclic nucleotide signals involved in signal transduction from the activation of FceR to particle fusion of vesicle rupture. cAMP in mast cells is involved in the regulation of the degranulation process, and is correlated with the physiological and pathological, cyclic chronic urticarial and treatment outcomes, which can stabilize the mast cell membrane and inhibit the release of allergic mediators $(25,26)$. PKA is a cAMP-dependent protein kinase, which can catalyze the phosphorylation of serine and threonine 
residues in proteins (27). It has been shown that the activity of PKA is affected in the process of mast cell degranulation, and certain drugs can inhibit the degranulation of mast cells, partly due to regulation of the activity of PKA $(28,29)$. In the present study, it was found that BCGE significantly reversed the OVA-induced reduction of cAMP and expression of PKA in mast cells, which suggested that BCGE suppressed mast cell degranulation by increasing the levels of cAMP and the expression of PKA.

In conclusion, the present study demonstrated that the intramuscular application of BCGE inhibited the development of OVA-induced anaphylaxis in vitro and in vivo. The inhibitory effect of BCGE was mediated by inhibiting IgE-mediated mast cell degranulation. The mechanism underlying this therapeutic effect of BCGE was mediated predominantly by a reduction in the level of IL-4, an increase in the level of IFN- $\gamma$ and the activation of cAMP-PKA. These results suggested that BCGE may offer potential as a therapeutic candidate for OVA-induced anaphylaxis.

\section{Acknowledgements}

This study was supported by a grant from the National Natural Science Foundation of the People's Republic of China (grant nos. 71273279 and 81501346).

\section{References}

1. Galli SJ, Tsai M and Piliponsky AM: The development of allergic inflammation. Nature 454: 445-454, 2008.

2. Simons FE: Anaphylaxis. J Allergy Clin Immunol 125 (2 Suppl 2): S161-S181, 2010

3. Choi EJ, Lee S, Hwang JS, Im SH, Jun CD, Lee HS and Kim SH: DA-9601 suppresses 2, 4-dinitrochlorobenzene and dust mite extract-induced atopic dermatitis-like skin lesions. Int Immunopharmacol 11: 1260-1264, 2011.

4. Furue M, Terao H, Rikihisa W, Urabe K, Kinukawa N, Nose Y and Koga T: Clinical dose and adverse effects of topical steroids in daily management of atopic dermatitis. Br J Dermatol 148: 128-133, 2003.

5. Leung DY, Jain N and Leo HL: New concepts in the pathogenesis of atopic dermatitis. Curr Opin Immunol 15: 634-638, 2003.

6. Galli SJ, Nakae S and Tsai M: Mast cells in the development of adaptive immune responses. Nat Immunol 6: 135-142, 2005.

7. Caramalho I, Lopes-Carvalho T, Ostler D, Zelenay S, Haury M and Demengeot J: Regulatory T cells selectively express toll-like receptors and are activated by lipopolysaccharide. J Exp Med 197: 403-411, 2003.

8. Yamamoto S, Kuramoto E, Shimada S and Tokunaga T: In vitro augmentation of natural killer cell activity and production of interferon-alpha/beta and -gamma with deoxyribonucleic acid fraction from Mycobacterium bovis BCG. Jpn J Cancer Res 79: 866-873, 1988

9. Yamamoto S, Yamamoto T, Kataoka T, Kuramoto E, Yano O and Tokunaga $\mathrm{T}$ : Unique palindromic sequences in synthetic oligonucleotides are required to induce IFN [correction of INF] and augment IFN-mediated [correction of INF] natural killer activity. J Immunol 148: 4072-4076, 1992.

10. Fujieda S, Iho S, Kimura Y, Sunaga H, Igawa H, Sugimoto C, Yamamoto $\mathrm{S}$ and Saito $\mathrm{H}$ : DNA from mycobacterium bovis bacillus Calmette-Guérin (MY-1) inhibits immunoglobulin E production by human lymphocytes. Am J Respir Crit Care Med 160: 2056-2061, 1999.

11. Cheng D, Zheng B, Chen W and Chen Z: The prophylactic effect of BCG polysaccharides nucleic acid on the acute attack of chronic obstructive pulmonary disease. Hua Xi Yi Ke Da Xue Xue Bao 33: 121-122, 2002 (In Chinese).
12. Xiong C, Li Q, Lin M, Li X, Meng W, Wu Y,Zeng X, Zhou H and Zhou G: The efficacy of topical intralesional BCG-PSN injection in the treatment of erosive oral lichen planus: A randomized controlled trial. J Oral Pathol Med 38: 551-558, 2009.

13. Deng T, Liu B, Duan X, Zhang T, Cai C and Zeng G: Systematic review and cumulative analysis of the combination of mitomycin C plus Bacillus Calmette-Guérin (BCG) for non-muscle-invasive bladder cancer. Sci Rep 7: 3172, 2017.

14. Jin YJ, Song ZY, Hu Y, Qian XB, Wang XY and He XY: Effects of montelukast and BCG-PSN on the expression of STAT5b mRNA and IL-4 mRNA in blood mononuclearcells of rats with asthma. Zhongguo Dang Dai Er Ke Za Zhi 11: 133-137, 2009 (In Chinese).

15. Zuo CX, Huang JH, Liao ZH, Lu JY and Chen J: Effects of BCG-PSN on serum levels of IL-4 and IL-12 in patients with condyloma acuminatum. Zhong Nan Da Xue Xue Bao Yi Xue Ban 29: 690-692, 2004 (In Chinese).

16. Sun M, Wang S, Zhao L, Zhao H, Yao W, Jin W and Wei M: Suppression of 2,4-dinitrochlorobenzene-induced atopic dermatitis by extract of Bacillus Calmette-Guerin. Mol Med Rep 9: 689-694, 2014.

17. Cho A and Seok SH: Ethical guidelines for use of experimental animals in biomedical research. J Bacteriol Virol 43: 18-26, 2013.

18. Finkelman FD: Anaphylaxis: Lessons from mouse models. J Allergy Clin Immunol 120: 506-515; quiz 516-517, 2007.

19. Inagaki $\mathrm{N}$ and Nagai $\mathrm{H}$ : Analysis of the mechanism for the development of allergic skin inflammation and the application for its treatment: Mouse models for the development of remedies for human allergic dermatitis. J Pharmacol Sci 110: 251-259, 2009.

20. Evora PR and Simon MR: Role of nitric oxide production in anaphylaxis and its relevance for the treatment of anaphylactic hypotension with methylene blue. Ann Allergy Asthma Immunol 99: 306-313, 2007.

21. Kumar P, Shen Q, Pivetti CD, Lee ES, Wu MH and Yuan SY: Molecular mechanisms of endothelial hyperpermeability: Implications in inflammation. Expert Rev Mol Med 11: e19, 2009.

22. Matsuoka H, Maki N, Yoshida S, Arai M, Wang J, Oikawa Y, Ikeda T, Hirota N, Nakagawa $\mathrm{H}$ and Ishii A: A mouse model of the atopic eczema/dermatitis syndrome by repeated application of a crude extract of house-dust mite Dermatophagoides farinae. Allergy 58: 139-145, 2003.

23. Kim SH, Jun CD, Suk K, Choi BJ, Lim H, Park S, Lee SH, Shin HY, Kim DK and Shin TY: Gallic acid inhibits histamine release and pro-inflammatory cytokine production in mast cells. Toxicol Sci 91: 123-131, 2006.

24. Kang JS, Yoon WK, Han MH, Lee H, Lee CW, Lee KH, Han SB, Lee K, Yang KH, Park SK and Kim HM: Inhibition of atopic dermatitis by topical application of silymarin in $\mathrm{NC} / \mathrm{Nga}$ mice. Int Immunopharmacol 8: 1475-1480, 2008.

25. Sullivan TJ, Parker KL, Kulczycki A Jr and Parker CW: Modulation of cyclic AMP in purified rat mast cells. III. Studies on the effects of concanavalin A and anti-IgE on cyclic AMP concentrations during histamine release. J Immunol 117: 713-716, 1976.

26. Kurosawa M, Mori H, Nagai $\mathrm{H}$ and Koda A: Change in the activity of the cyclic AMP-dependent protein kinase in antigen-stimulated sensitized mast cells and effect of drugs inhibiting allergic mediator release. Jpn J Pharmacol 43: 454-457, 1987.

27. Kurosawa M: Studies of phosphorylation in rat mast cells (sixth report). Cyclic AMP-dependent protein phosphorylation in rat mast cell granule membranes. Nihon Yakurigaku Zasshi 86: 87-92, 1985 (In Japanese).

28. Sullivan TJ, Parker KL, Eisen SA and Parker CW: Modulation of cyclic AMP in purified rat mast cells. II. Studies on the relationship between intracellular cyclic AMP concentrations and histamine release. J Immunol 114: 1480-1485, 1975.

29. Lau HY and Chan CL: Modulation of intracellular cyclic AMP in immunologically activated rat peritoneal mast cells by prostaglandin D2. Inflamm Res 50 (Suppl 2): S61-S62, 2001. 